\title{
Establishing CRISPR/Cas9 in Lipomyces starkeyi
}

Zoe Lau', Bonnie Mcneil ${ }^{1}$, David Stuart ${ }^{1}$

${ }^{1}$ Department of Biochemistry, University of Alberta

\begin{abstract}
The goal of this project was to adapt the Yarrowia lipolytica plasmid based CRISPR/Cas9 system for usage in Lipomyces starkeyi. Lipomyces starkeyi is an oleaginous yeast, which synthesizes and stores high amounts of intracellular lipids. This specific yeast can store lipids at concentrations higher than $60 \%$ of its dry cell weight. Due to these high concentrations of lipids, L. starkeyi is a desired organism for the production of biofuels and other oleochemicals. However, there is a lack of knowledge and of genetic tools when trying to engineer the cells to produce these lipids for our use. The genome editing tool, CRISPR/Cas9 is efficient and simple, therefore desirable for the engineering of $L$. starkeyi. The goal was achieved by replacing the $\mathrm{Y}$. lipolytica promoter with a L. starkeyi promoter, inserting guide RNA, as well as confirming cas9 protein expression.
\end{abstract}

Key words:

CRISPR, Lipomyces starkeyi, Lipomyces starkeyi CRISPR, gene editing, biofuels, Lipomyces starkeyi biofuels,

Cite as: Lau, Z., Mcneil, B., Stuart, D. 2019. Establishing CRISPR/Cas9 in Lipomyces starkeyi . Alberta Academic Review, Vol 2 (2) 51-52, WISEST Special Issue (non peer-reviewed), DOI 10.29173/aar61. 
Lau et al., 2019

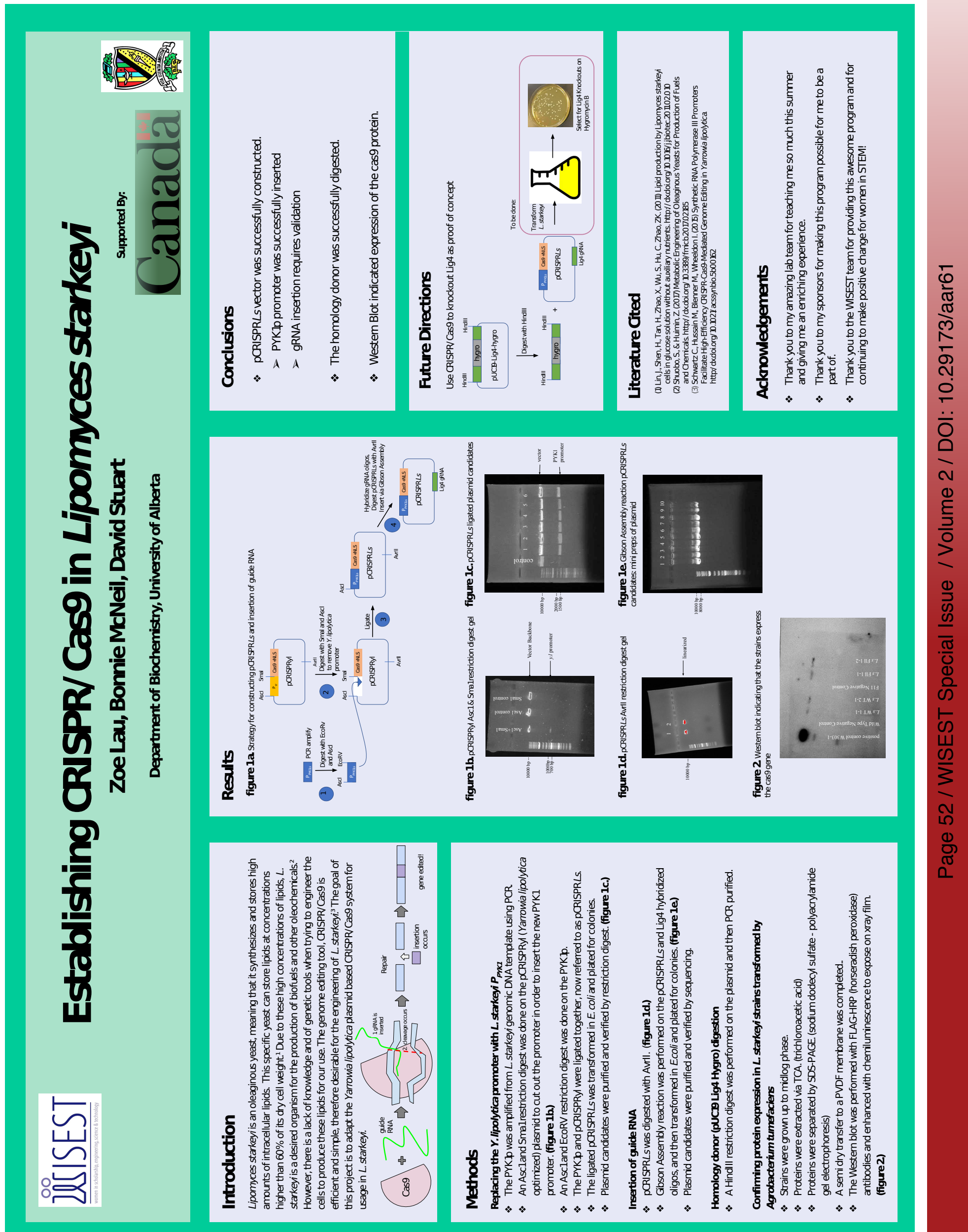

